\title{
Apparent duration and numerosity as a function of melodic familiarity
}

\author{
KATHLEEN H. KOWAL \\ University of North Carolina at Wilmington, Wilmington, North Carolina
}

\begin{abstract}
Measurements of the apparent duration, numerosity, familiarity, predictability, and organization of musical sequences were obtained to resolve conflicting claims about the effects of stimulus variables on duration judgments and to assess whether subjective number is an effective mediating variable underlying duration judgments. Intervals filled with perceptually familiar, organized, and predictable sequences of musical notes were judged by verbal estimation and, in most cases, by magnitude estimation to be longer and to have more notes than their perceptually unfamiliar reverse counterparts. The relationship between perceived and physical duration or number is consistent with Stevens's law, with exponents typical of reported values. Temporal and numerosity exponents derived from verbal estimates, but not from magnitude estimates, were higher for familiar, organized, and predictable sequences, but only when sequences were not paired with their reverse counterparts. Exponents and intercepts were not independent, but were inversely related; both may be needed to describe the duration and numerosity data.
\end{abstract}

Duration perception is affected by several factors. Time intervals containing complex, unfamiliar, more numerous, or less predictable stimuli are estimated to be longer than intervals containing simpler (e.g., Block, 1978, Experiment 2; Ornstein, 1969; Schiffman \& Bobko, 1974), more familiar (e.g., Avant \& Lyman, 1975; von Sturmer, 1966), more predictable (e.g., Frankenhaeuser, 1959; Ornstein, 1969), or fewer stimuli (e.g., Buffardi, 1971; Fraisse, 1963; Frankenhaeuser, 1959; Ornstein, 1969; Poynter \& Homa, 1983; Schiffman \& Bobko, 1977).

Several attractive cognitive theories have been offered to explain these findings. For example, Ornstein (1969) argued that apparent duration varies with the storage space occupied by the interval's content and that more organized and less complex stimuli occupy less space. Others take the amount of material remembered (Frankenhaeuser, 1959), the perceived number of events (Fraisse, 1963), or the amount of information processing (Michon, 1965) to determine apparent duration. Some time-perception studies are consistent with one or more of these theories. Others are contradictory: Estimates of duration are sometimes not a monotonic function of events' complexity (Block, 1974, Experiment 2, 1978, Experiment 1; Bobko, Schiffman, Castino, \& Chiappetta, 1977; Hogan, 1975; Mo \& Michalski, 1972; M. J. Smith, 1975; N. C. Smith, Jr., 1969), number (E. C. Jones \& Natale, 1973), predictability (Kowal, 1981), or familiarity (Kowal, 1976; Schiffman \& Bobko, 1977; Thomas \& Weaver, 1975).

\footnotetext{
A preliminary report of these data was presented at a conference on timing and time perception held May 10-13, 1983, by the New York Academy of Sciences (Kowal, 1984). I thank Julian Hochberg, Martha Teghtsoonian, and the anonymous reviewers for their suggestions and comments on the manuscript. The author's mailing address is: Psychology Department, University of North Carolina at Wilmington, Wilmington, NC 28403-3297.
}

Procedural and methodological contaminants, such as (1) method of measurement, (2) whether subjects perceive the experimental manipulation of events within the interval, (3) whether judgments of one duration or of several durations are obtained, and (4) whether the same subjects make several judgments under different stimulus conditions or under only one stimulus condition, may be responsible for the unreliability of time-estimation results. However, none of these has been studied extensively enough to assess its effect on time judgments.

The present research measured the effects of two levels of three subjective dimensions-familiarity, organization, and predictability - on two related measures of the apparent duration of a large number of note sequences of different durations. Measures of apparent numerosity were also obtained for the same note sequences. Half of the stimuli were very familiar simple melodies; the others were the same melodies played backward, assumed to be unfamiliar and lower in subjective organization. Subjective duration and numerosity were measured separately, by magnitude estimation with some subjects and by verbal estimation with others.

Stevens's power law $\left(S=a R^{b}\right)$ has been proposed to describe the psychophysical relation between duration and estimated duration (S. S. Stevens, 1957). That law gives two parameters, the exponent $b$ and the multiplicative constant $a$, or the slope $b$ and intercept $\log a$ of the logarithmic transformation. The temporal exponent, which has been used to measure the growth of apparent duration relative to physical duration (Carlson \& Feinberg, 1968), ranges from .53 to 1.55 (Allan, 1983; Eisler, 1976; S. S. Stevens \& Galanter, 1957; S. S. Stevens \& Greenbaum, 1966). The intercept, believed by some to be a scaling factor, either arbitrary (Gescheider, 1976, p. 127) or private and individual (Zwislocki \& Goodman, 1980), is rarely analyzed or reported. 
To serve as a useful summary description of the scaling of apparent duration, a psychophysical function should be sensitive to those factors that affect the time judgments themselves, and exponents have shown such sensitivity for stimulus attributes other than duration: The exponent for heaviness depends, in part, on volume (J. C. Stevens \& S. S. Stevens, 1963); that for loudness, on the degree of masking (S. S. Stevens, 1966); and that for tactual roughness, on motivation level (Goldner, Reuder, Riba, \& Jarmon, 1971). However, the temporal exponent seems to be unaffected by stimulus familiarity or by the amount of material recalled (Kowal, 1976), although sometimes it is affected by stimulus predictability (Kowal, 1981). If the familiarity, organization, and predictability of stimulus sequences affect time judgments, such effects should be reflected in temporal exponents and/or multiplicative constants, if the power function is to be a useful description, and also should be reflected in the parameters of the power function for apparent numerosity, if the latter mediates duration judgments, as has been proposed (Fraisse, 1963).

\section{EXPERIMENT 1}

\section{Method}

Subjects. One hundred and eight undergrduate students from classes in introductory psychology at the University of North Carolina at Wilmington participated to fulfill a course requirement.

Materials. Seventeen musical note sequences, generated on an electronic synthesizer and recorded on a reel-to-reel tape recorder, were melodies assumed to be familiar to the subjects (e.g., "Dixie," "America the Beautiful"), and 17 were the same sequences in reverse order, made by reversing the reels. Cassette recordings were then made of both forward and reverse sequences. Sequences ranged from 13.5 to $48 \mathrm{sec}$ and from 23 to 130 notes.

Procedure. The subjects were assigned randomly to one of four judgment conditions--magnitude estimation (24 subjects) and verbal estimation (34 subjects) of sequence duration, and magnitude estimation (28 subjects) and verbal estimation (22 subjects) of number of notes. In making magnitude estimates, the subjects were cautioned not to estimate actual numbers of seconds or notes, but to select a number whose magnitude represented the perceived magnitude of duration or numerosity. If, for example, they called the first sequence 100 and the second seemed to be twice as long or to contain twice as many notes, they were to assign it the number 200. They were also told to use any numbers they chose except for 0 or negative numbers. The verbal estimation task required the subjects to estimate sequence durations in minutes and seconds or to estimate the actual number of notes.

In all four judgment conditions, the subjects listened to 17 forward and 17 reverse sequences, presented in two orders, with roughly half the subjects receiving each order in each condition. In Order 1, the first and last stimuli were neither the largest nor the smallest (in duration or in number of notes), and each sequence was followed by its forward or reverse counterpart, with the order of the sequence pairs randomly determined. Order 2 was the reverse of Order 1.

Tested in groups of from 1 to 6 , the subjects placed their watches face down on the table. After each stimulus sequence, they were asked first to estimate duration or numerosity and then to rate each sequence from 1 to 7 on each of three dimensions-familiarity, organization, and predictability - with 1 representing least and 7 most of an attribute. Dimensions to be judged were illustrated by words and sentences that varied in familiarity, organization, and predictability (e.g., random words vs. familiar sentences). Each subject wrote all responses on one page, and all responses were available to the subject's view throughout the experiment.

\section{Results}

Ratings on familiarity, organization, and predictability. In all four judgment conditions, mean ratings of forward sequences (ranging from 6.96 to 6.98 on familiarity, from 6.59 to 6.77 on organization, and from 6.88 to 6.96 on predictability) were significantly greater than those of reverse sequences (ranging from 1.69 to 1.81 , from 2.87 to 3.62 , and from 2.29 to 2.38 on the three dimensions, respectively; $t$ tests yielded $p<.001$ for comparisons of means within each condition).

Data transformations. Throughout Experiments 1, 2, and 3, stimulus and/or response values are sometimes log transformed, depending on the nature of the data and on the data analysis. Table 1 outlines the conditions under which log transforms are performed.

Estimates of duration and number. Means of the subjects' (normally distributed) verbal estimates of duration and of number, collapsed together across orders, were calculated for each duration and number. Two durations and three numerosities were represented by more than one melody; these were averaged, yielding estimates of 15 different durations and 14 different numerosities. Geometric means of all subjects' magnitude estimates were also determined for each duration and number. The top panels of Figures 1 and 2 show mean duration and numerosity estimates, respectively; the top panels of Figures 3 and 4 show geometric means of duration and numerosity estimates, respectively, in logarithmic coordinates. Geometric means were used to normalize the skewed distributions of magnitude estimates.

Four separate two-way analyses of variance (ANOVAs) of the verbal estimates or of the $\log _{10}$ magnitude estimates, with repeated measures on sequence direction and duration or number, were performed. In all cases, forward sequences were judged to be longer and to have more notes than reverse sequences. However, the interpretation of these effects must take into account the reliable interactions between sequence direction and duration or

Table 1

Form of Stimulus and Response Variables for Verbal Estimation and Magnitude Estimation Under the Different Data Presentations and Analyses

\begin{tabular}{|c|c|c|}
\hline \multirow[b]{2}{*}{ Data Presentation } & \multicolumn{2}{|c|}{ Variable } \\
\hline & Stimulus & Response \\
\hline $\begin{array}{l}\text { Analyses of Variance } \\
\text { Verbal Estimates } \\
\text { Magnitude Estimates }\end{array}$ & $\begin{array}{l}\text { Untransformed } \\
\text { Untransformed }\end{array}$ & $\begin{array}{l}\text { Untransformed } \\
\log _{10}\end{array}$ \\
\hline $\begin{array}{l}\text { Figures } \\
1 \text { and } 2 \text { (Verbal Estimates) } \\
3 \text { and } 4 \text { (Magnitude Estimates) }\end{array}$ & $\begin{array}{l}\text { Untransformed } \\
\log _{10}\end{array}$ & $\begin{array}{l}\text { Untransformed } \\
\log _{10}\end{array}$ \\
\hline $\begin{array}{l}\text { Power Functions Fitted } \\
\text { Verbal Estimates } \\
\text { Magnitude Estimates } \\
\end{array}$ & $\begin{array}{l}\log _{10} \\
\log _{10}\end{array}$ & $\begin{array}{l}\log _{10} \\
\log _{10}\end{array}$ \\
\hline
\end{tabular}




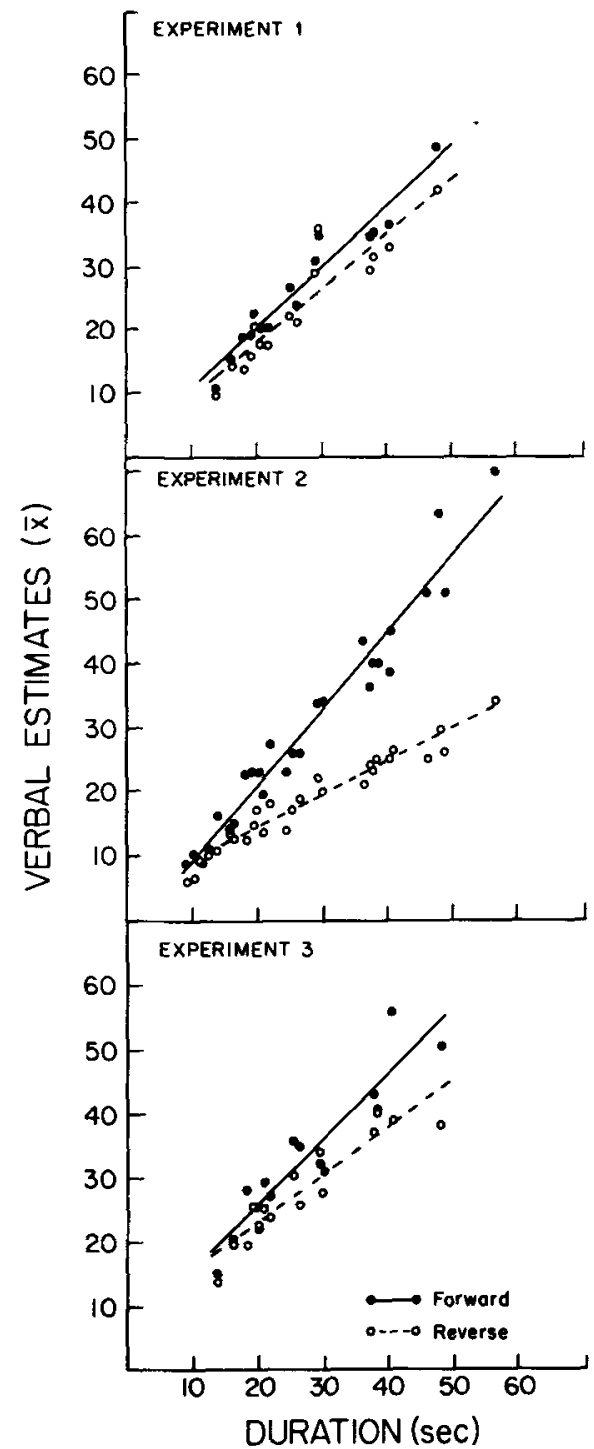

Figure 1. Mean verhal estimates of the durations of forward (filled circles) and reverse (open circles) melodic sequences. In Experiments 1 and 3, the subjects estimated both forward and reverse sequences; in Experiment 2, the subjects extimated only forward or only reverse sequences. (Lines fitted by least squares method.)

numerosity found in all conditions, with a pattern suggesting that there are slope differences for verbal estimates but not for magnitude estimates. The effect of sequence direction was reliable for verbal estimates of duration $[F(1,33)=47.15, p<.001]$ and numerosity $[F(1,21)$ $=57.23, p<.001]$ and for $\log$ magnitude estimates of duration $[F(1,23)=15.04, p<.001]$ and numerosity $[F(1,27)=33.69, p<.001]$. As duration or numerosity increased, verbal estimates and log magnitude estimates increased $(F \mathrm{~s}$ were significant at $p<.001)$. Interactions were significant for verbal estimates $[F(14,462)=1.76$, $p<.05]$ and $\log$ magnitude estimates $[F(14,322)=2.54$, $p<.002]$ of duration and for verbal estimates $[F(13,273)$ $=3.85, p<.001 \mathrm{~J}$ and $\log$ magnitude estimates
$[F(13,351)=2.15, p<.01]$ of numerosity. Tests of simple main effects showed that duration estimates of forward sequences were significantly greater than those of reverse sequences by verbal estimation at durations of 18 , $25,37.5,38,40.5$, and $48 \mathrm{sec}$ and by magnitude estimation at $19.5 \mathrm{sec}$ only; numerosity estimates of forward sequences were significanty greater than those of reverse sequences by verbal estimation for numerosities of 46 , $68,86,113$, and 130 notes and by magnitude estimation for numerosities of $33,41,46$, and 68 notes. The top panels of Figures 1, 2, 3, and 4 show that the reliable interactions, which appear as slope differences for the untransformed verbal estimates, are not apparent for the log transformed magnitude estimates. Although these interactions suggest that group slopes derived from all subjects' estimates of forward and reverse sequences differ, at least for the verbal estimates, an analysis of the individual functions derived for each subject indicates otherwise.

Exponents and intercepts derived from estimates. To examine the effect of sequence direction on parameters

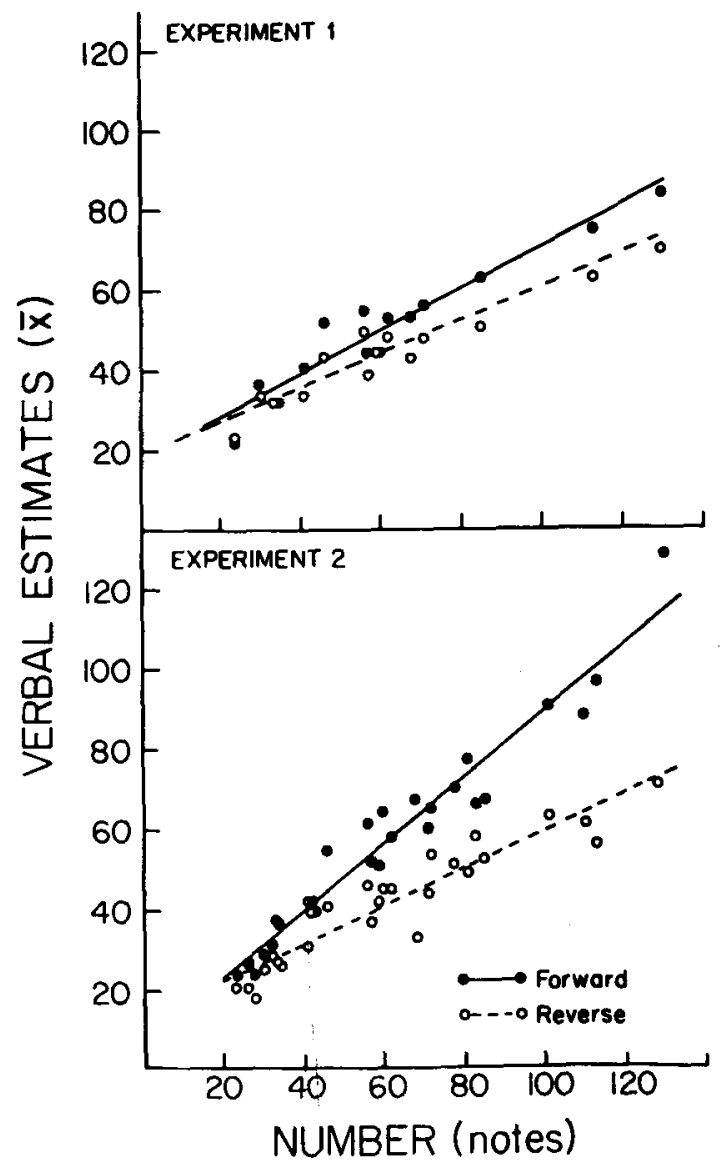

Figure 2. Mean verbal estimates of the number of notes occurring within forward (filled circles) and reverse (open circles) melodic sequences. In Experiment 1, the subjects estimated both forward and reverse sequences; in Experiment 2, the subjects estimated only forward or only reverse sequences. (Lines fitted by least squares method.) 
of Stevens's power law $\left(S=a R^{b}\right)$, measures of the exponent $b$ (or slope in logarithmic coordinates) and the logarithm of the scaling factor $a$ (or intercept in logarithmic coordinates) were obtained from estimates of duration and numerosity. Individual slopes (exponents), intercepts (log scaling factors), and correlation coefficients of best-fitting straight lines relating $\log _{10}$ of perceived duration or numerosity to $\log _{10}$ of actual duration or number of notes were determined for each subject by the least squares method. The means of these values and the standard errors for mean exponents are presented separately in Table 2 for forward and reverse sequences. Mean values for correlation coefficients, determined by the use of $z$ scores, were significant.

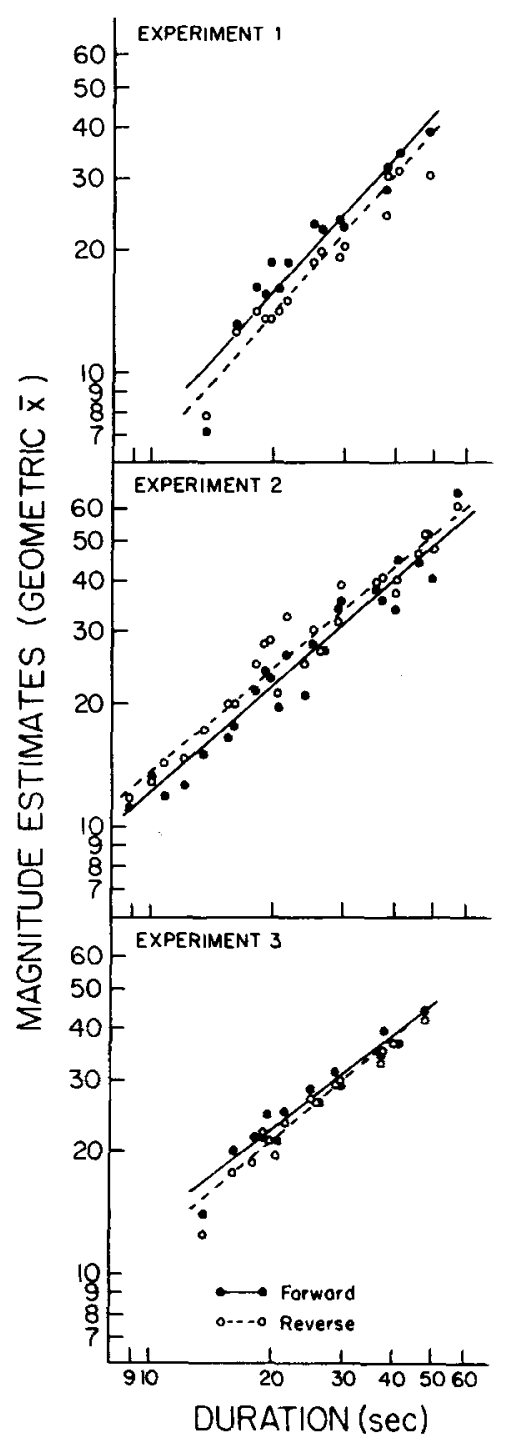

Figure 3. Geometric means of magnitude estimates of the durations of forward (filled circles) and reverse (open circles) melodic sequences, plotted in log-log coordinates. In Experiments 1 and 3, the subjects estimated both forward and reverse sequences; in Experiment 2 , the subjects estimated only forward or only reverse sequences. (Lines fitted by least squares method.)

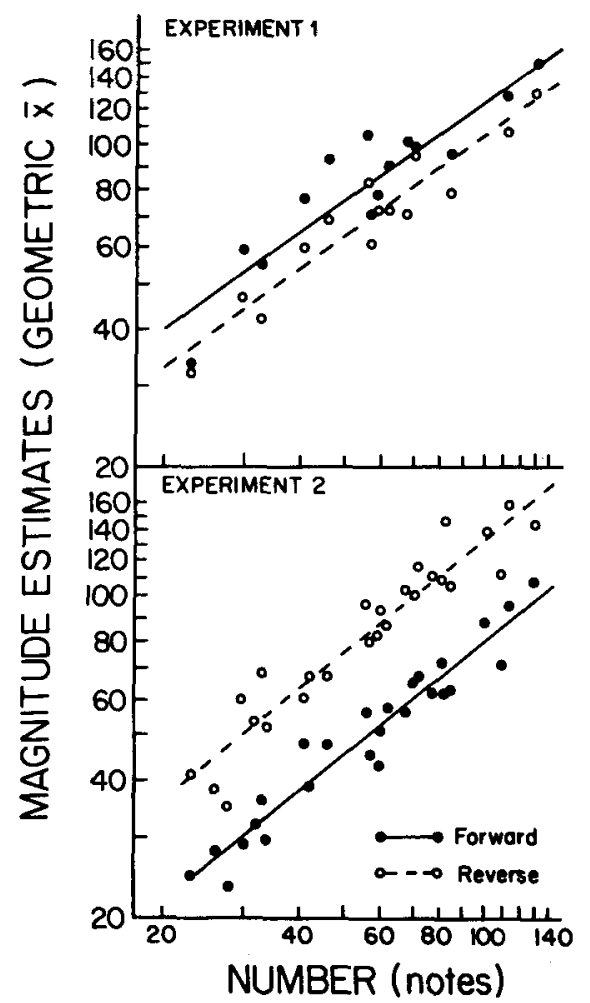

Figure 4. Geometric means of magnitude estimates of the number of notes occurring within forward (filled circles) and reverse (open circles) melodic sequences, plotted in log-log coordinates. In Experiment 1, the subjects estimated both forward and reverse sequences; in Experiment 2, the subjects estimated only forward or only reverse sequences. (Lines fitted by least squares method.)

Temporal exponents for forward and reverse sequences did not differ either by verbal estimation $[t(33)=.86$, $p>.10]$ or by magnitude estimation $[t(23)=1.61$, $p>.10$ ]. Similarly, numerosity exponents of forward and reverse sequences did not differ by magnitude estimation $[t(27)=.14, p>.8]$, but were greater for forward sequences by verbal estimation $[t(21)=3.39$, $p<.01]$. The pattern of results was similar for the intercepts: Sequence direction did not affect intercepts derived from verbal estimates $[t(33)=1.75, p>.05]$ or magnitude estimates $[t(23)=.65, p>.1]$ of duration or from magnitude estimates of numerosity $[t(27)=1.63$, $p>.05$ ], but intercepts derived from verbal estimates of numerosity were significantly higher for reverse sequences $[t(21)=2.47, p<.05]$.

Additional analyses support the finding that exponents did not differ:

1. Eliminating from the analyses those functions with low correlations (i.e., $r^{2}<.80$; see Teghtsoonian \& Teghtsoonian, 1978) showed no reliable differences between exponents for forward and reverse sequences $(t$ tests yielded $p>.05$ ).

2. Although linear functions, determined for each subject, relating verbal estimates of duration to duration itself fit the data at least as well as power functions (mean 
Table 2

Means of the Individual Exponents (and Standard Errors, SEs), Intercepts (Log Scaling Factors), and Correlation Coefficients Derived From Each Subject's Verbal Estimates or From Each Subject's Magnitude Estimates of Duration and of Numerosity (Repeated Measures)

\begin{tabular}{|c|c|c|c|c|}
\hline \multirow[b]{3}{*}{ Method } & \multicolumn{4}{|c|}{ Sequence } \\
\hline & \multicolumn{2}{|c|}{ Forward } & \multicolumn{2}{|c|}{ Reverse } \\
\hline & Mean & $S E$ & Mean & $S E$ \\
\hline \multicolumn{5}{|c|}{ Duration } \\
\hline \multicolumn{5}{|c|}{ Magnitude Estimation } \\
\hline Exponent & 1.08 & .07 & 1.01 & .07 \\
\hline Intercept & -.21 & & -.17 & \\
\hline$r$ & .81 & & .78 & \\
\hline \multicolumn{5}{|c|}{ Verbal Estimation } \\
\hline Exponent & 1.02 & .05 & 1.06 & .05 \\
\hline Intercept & -.08 & & -.19 & \\
\hline$r$ & .81 & & .82 & \\
\hline \multicolumn{5}{|c|}{ Numerosity } \\
\hline \multicolumn{5}{|c|}{ Magnitude Estimation } \\
\hline Exponent & .70 & .05 & .70 & .06 \\
\hline Intercept & 1.26 & & 1.05 & \\
\hline$r$ & .86 & & .80 & \\
\hline \multicolumn{5}{|c|}{ Verbal Estimation } \\
\hline Exponent & .66 & .04 & .56 & $.04 *$ \\
\hline Intercept & .51 & & $.64^{*}$ & \\
\hline$r$ & .80 & & .75 & \\
\hline
\end{tabular}

${ }^{*}$ Difference between forward and reverse sequences is significant.

$r s$ ranged from .80 to .82$)$, sequence direction had no effect on slopes of individual linear functions $[t(33)=1.86$, $p>.05]$.

3. Combining exponents derived from magnitude and verbal estimates yielded no reliable effect of sequence direction on temporal $[t(57)=.21, p>.5]$ or numerosity $[t(49)=1.72, p>.05]$ exponents.

Although neither exponents nor intercepts were consistently affected by sequence direction, they differed for forward and reverse sequences. When subjects were separated into categories depending on whether the intercept was higher for forward sequences or for reverse sequences, the exponents of the 108 subjects who had provided repeated measures showed two reliable clusters ( $t$ tests yielding $p<.01$ for all comparisons): For 52 subjects, exponents were larger and intercepts smaller for forward sequences; for the 56 subjects in the other cluster, the reverse was true.

Individual exponents and intercepts were inversely related for magnitude estimates of duration [forward: $r(22)$ $=-.58, p<.01 ;$ reverse: $r(22)=-.64, p<.01]$ and numerosity [forward: $r(26)=-.69, p<.001$; reverse: $r(26)=-.70, p<.001]$, as were those for verbal estimates of duration [forward: $r(32)=-.94, p<.001$; reverse: $r(32)=-.96, p<.001]$ and numerosity [forward: $r(20)=-.96, p<.001$; reverse: $r(20)=-.97$, $p<.001]$.

The exponents may have been insensitive to familiarity in this experiment, because the subjects judged both forward and reverse versions and their judgments may have been mutually contaminated. In Experiment 2, there- fore, subjects judged only forward or only reverse versions of melodic sequences, and the number of sequences was increased from 17 to 35 .

\section{EXPERIMENT 2}

\section{Method}

Subjects. One hundred and twenty-seven introductory psychology students participated to fulfill a course requirement.

Materials. The 34 musical sequences of Experiment 1 were supplemented by 36 similar sequences, for a total of $\mathbf{3 5}$ familiar melodies and 35 reverse sequences. Duration ranged from 8.5 to $56.5 \mathrm{sec}$, and number of notes ranged from 23 to 130 .

Procedure. The subjects were assigned randomly to one of four judgment conditions-magnitude estimation (57 subjects) and verbal estimation (20 subjects) of sequence duration and magnitude estimation ( 30 subjects) and verbal estimation (20 subjects) of number of notes within the sequence. In all judgment conditions, approximately equal numbers of subjects judged the 35 forward sequences and the 35 reverse sequences. In the magnitude-estimationof-duration condition, each of three random orders of the 35 sequences was presented to roughly one third of the subjects. In the other three judgment conditions, only one order of sequences was used.

Ratings of all sequences (from 1 to 7) were obtained from all subjects on the dimensions of familiarity, predictability, and organization. All other instructions and testing conditions were the same as in Experiment 1.

\section{Results}

Ratings on familiarity, organization, and predictability. In all four judgment conditions, mean ratings of forward sequences (ranging from 6.05 to 6.15 on familiarity, from 6.03 to 6.25 on organization, and from 5.81 to 6.07 on predictability) were reliably greater than those of reverse sequences (ranging from 2.00 to 2.85 , from 3.48 to 3.64 , and from 2.94 to 3.20 on the three dimensions, respectively; $t$ tests yielded $p<.001$ for comparisons of means within each condition and for each sequence).

Estimates of duration and number. The means of all subjects' verbal estimates of duration and of number were calculated for each duration and for each numerosity of forward and reverse sequences separately. Seven durations and four numerosities were represented by more than one melody; the multiple estimates were averaged, which resulted in estimates for 28 different durations and 27 different numerosities. Geometric means of all subjects' magnitude estimates were similarly determined for each duration and numerosity. The middle panels of Figures 1 and 2 show the mean estimates of duration and number; the middle panels of Figures 3 and 4 show the geometric means of the estimates of duration and number in log-log coordinates.

Four separate two-way ANOVAs were performed on the verbal estimates or the logarithms of the magnitude estimates, with duration or number as the within-subjects factor and sequence direction as the between-subjects factor. With respect to verbal estimates, the results of this analysis parallel those of Experiment 1: Forward sequences were judged to be longer $[F(1,18)=9.86$, $p<.01]$ and to have more notes $[F(1,18)=8.28$, 
$p<.01]$; the effects of duration and numerosity were reliable $(p s<.01)$; the reliable interactions between sequence direction and duration $[F(26,468)=5.34$, $p<.01]$ or numerosity $[F(25,450)=4.78, p<.01]$ suggest that group slopes differ. Tests of simple main effects showed that verbal estimates of forward sequences were reliably greater than those of reverse sequences for all durations of $29 \mathrm{sec}$ and longer and for numerosities of $56,60,68,78,81,101$, and greater. With magnitude estimates, the results are idiosyncratic: Log magnitude estimates of duration were not affected by sequence direction $[F(1,55)=.08, p>.25]$, but those of numerosity were greater for reverse than for forward sequences $[F(1,28)=6.65, p<.05]$; the effects of duration and numerosity continued to be reliable ( $p s<.01$ ); there were no reliable interactions between sequence direction and duration or numerosity ( $p s>.10$ ). These discrepant results are not surprising. With magnitude estimation and no specified modulus and standard, a between-subjects design is, at best, not a sensitive test and, at worst, no test at all of differences in the levels of the forward and reverse functions-unlike Experiment 1, with its withinsubjects design. The effect of sequence direction, which might have been concealed by the self-selection of different standards and moduli by the subjects in the forward and reverse conditions, should emerge when magnitude estimates are transformed to take into account this selfselection process.

The robust effect of sequence direction, which occurred for verbal estimates here and for all estimates in Experiment 1, also occurred for magnitude estimates of duration when they were transformed. Each subject's estimates were divided by his/her estimate of the third sequence $(15.5 \mathrm{sec})$, which was used as the reference because it was the same duration for all three stimulus orders. An ANOVA of the transformed estimates showed that forward sequences were judged to be longer $[F(1,55)=7.96$, $p<.01]$, that the effect of duration was reliable $[F(26,1430)=79.71, p<.01]$, and that the interaction between the two factors was significant $[F(26,1430)=$ $4.08, p<.01]$. Tests of simple main effects showed that forward sequences were judged to be significantly longer than reverse sequences at durations of $25 \mathrm{sec}$ and longer, with the exception of the $40-\mathrm{sec}$ duration. ANOVAs of similarly transformed magnitude estimates of numerosity (the third, or reference, sequence contained 23 notes) showed that sequence direction had no effect on transformed estimates $[F(1,28)=.07, p>.25]$, that the effect of number was reliable $[F(25,700)=20.99$, $p<.01]$, and that the interaction between the two factors was not significant $[F(25,700)=1.13, p>.25]$.

Exponents and intercepts derived from estimates. Means of the exponents, intercepts, and correlation coefficients for each subject, derived as in Experiment 1, are presented with the standard errors for the mean exponents in Table 3. These data fit the psychophysical function with significant correlation coefficients.
Table 3

Means of the Individual Exponents (and Standard Errors, SEs) Intercepts (Log Scaling Factors), and Correlation Coefficients Derived From Each Subject's Verbal Estimates or From Each Subject's Magnitude Estimates of Duration and of Numerosity (Independent Design)

\begin{tabular}{|c|c|c|c|c|}
\hline \multirow[b]{3}{*}{ Method } & \multicolumn{4}{|c|}{ Sequence } \\
\hline & \multicolumn{2}{|c|}{ Forward } & \multicolumn{2}{|c|}{ Reverse } \\
\hline & Mean & $S E$ & Mean & $S E$ \\
\hline \multicolumn{5}{|c|}{ Duration } \\
\hline \multicolumn{5}{|c|}{ Magnitude Estimation } \\
\hline Exponent & .88 & .04 & .80 & .04 \\
\hline Intercept & .20 & & .35 & \\
\hline$r$ & .85 & & .82 & \\
\hline \multicolumn{5}{|c|}{ Verbal Estimation } \\
\hline Exponent & 1.08 & .08 & .81 & $.05^{*}$ \\
\hline Intercept & -.06 & & .04 & \\
\hline \multirow[t]{2}{*}{$r$} & .89 & & .84 & \\
\hline & \multicolumn{4}{|c|}{ Numerosity } \\
\hline \multicolumn{5}{|c|}{ Magnitude Estimation } \\
\hline Exponent & .81 & .06 & .76 & .07 \\
\hline Intercept & .27 & & $.60^{*}$ & \\
\hline$r$ & .81 & & .68 & \\
\hline \multicolumn{5}{|c|}{ Verbal Estimation } \\
\hline Exponent & .89 & .04 & $.69^{*}$ & .06 \\
\hline Intercept & .15 & & .33 & \\
\hline$r$ & .91 & & .81 & \\
\hline
\end{tabular}

*Difference between forward and reverse sequences is significant.

Exponents derived from verbal estimates of forward sequences were greater than those of reverse sequences for duration $[t(18)=2.85, p<.02]$ and for numerosity $[t(18)=2.73, p<.02]$. Sequence direction had no effect on exponents derived from magnitude estimates of duration $[t(55)=1.62, p>.05]$ or number $[t(28)=.58$, $p>.05]$; it also had no effect on intercepts derived from verbal estimates of duration $[t(18)=1.51, p>.10]$ and numerosity $[t(18)=2.09, p>.05]$ or on those derived from magnitude estimates of duration $[t(55)=.24$, $p>.05]$. Intercepts derived from magnitude estimates of numerosity were higher for reverse sequences $[t(28)$ $=2.04, p=.05]$.

Exponents and intercepts (log scaling factors) were inversely related for magnitude estimates of duration [reverse: $r(27)=-.40, p<.05$ ] and of numerosity [forward: $r(13)=-.76, p<.01$; reverse: $r(13)=$ $-.92, p<.01$ ], and for verbal estimates of duration [forward: $r(8)=-.92, p<.01$; reverse: $r(8)=-.88$, $p<.01$ ] and of numerosity [forward: $r(8)=-.95$, $p<.01$; reverse: $r(8)=-.76, p<.05$ ]. For magnitude estimates of the duration of forward sequences, this relationship approached significance $[r(27)=-.32$, $p<.10]$.

A third experiment was undertaken to address the possibility that some of the main results described are attributable to an interaction between subjects' two tasks (i.e., judging the attribute of duration or numerosity and rating sequences on familiarity, organization, and predict- 
ability). In Experiment 3, subjects either estimated the duration of sequences or rated sequences on one of the three dimensions, but did not do both.

\section{EXPERIMENT 3}

\section{Method}

Subjects. The subjects were 68 introductory psychology students who participated to fulfill a course requirement.

Materials. The 34 musical sequences of Experiment 1 were used here.

Procedure. Fourteen subjects were assigned randomly to one of two duration judgment conditions-magnitude estimation (8 subjects) and verbal estimation (6 subjects)-with no further ratings obtained. Fifty-four subjects were assigned randomly to rate the familiarity (18 subjects), the organization (18 subjects), or the predictability (18 subjects) of sequences, but not duration or numerosity. Ratings of from 1 to 7 represented the least (1) to most (7) of an attribute. Subjects in all conditions judged or rated the 17 pairs of forward and reverse sequences of Experiment 1. Sequences were presented in Order 1 (Experiment 1) for the duration judgment conditions and in Orders 1 and 2 (Experiment 1) for the rating conditions, with half the subjects assigned to each order.

\section{Results}

Ratings on familiarity, organization, and predictability. In all three rating conditions, mean ratings for forward sequences (6.94 on familiarity, 6.62 on organization, and 6.84 on predictability) were significantly greater than those for reverse sequences $(1.61,2.74$, and 2.89 , respectively; $t$ tests yielded $p<.001$ for comparisons of means within each rating condition).

Estimates of duration. Means of all subjects' verbal estimates (Figure 1, bottom panel) and geometric means of all subjects' magnitude estimates (Figure 3, bottom panel) were calculated for each duration, as in Experiment 1. A comparison of the geometric means and of the means showed that forward sequences were judged to be longer by both magnitude estimation $[t(16)=3.78$, $p<.01]$ and verbal estimation $[t(16)=3.08, p<.01]$.

Exponents and intercepts derived from estimates. As in Experiment 1, exponents, intercepts, and correlation coefficients were derived from the logarithms of each subject's estimates. Table 4 presents the means of these values. Exponents of forward and reverse sequences did not differ for magnitude estimation $[r(7)=.99, p>.30]$ or verbal estimation $[t(5)=.90, p>.40]$. Intercepts also showed no reliable effect of sequence direction ( $t$ tests yielded $p s>, 20$ ). Correlations between exponents and intercepts were negative and significant $(p<.02)$ for both methods and sequence directions. With the data of Experiments 1 and 3 combined, sequence direction had no effect on exponents or on intercepts derived from estimates by either method $(t$ tests yielded $p s>.05$ in each case).

These results show that the strong and reliable differences between duration or numerosity estimates of forward and reverse sequences were not attributable to the process of rating sequences with respect to familiarity, organization, and predictability, and that rating differences of forward and reverse sequences along the three dimen-
Table 4

Means of the Individual Exponents (and Standard Errors, SEs), Intercepts (Log Scaling Factors), and Correlation Coefficients Derived From Each Subject's Magnitude Fstimates or From Each Subject's Verbal Estimates of Duration (Repeated Measures-No Ratings)

\begin{tabular}{|c|c|c|c|c|}
\hline & \multicolumn{4}{|c|}{ Sequence } \\
\hline & \multicolumn{2}{|c|}{ Forward } & \multicolumn{2}{|c|}{ Reverse } \\
\hline & Mean & $S E$ & Mean & $S E$ \\
\hline \multicolumn{5}{|c|}{ Magnitude Estimation } \\
\hline Exponent & .77 & .11 & .84 & .17 \\
\hline Intercept & .35 & & .22 & \\
\hline$r$ & .84 & & .85 & \\
\hline \multicolumn{5}{|c|}{ Verbal Estimation } \\
\hline Exponent & .82 & .10 & .72 & .05 \\
\hline Intercept & .30 & & .33 & \\
\hline$r$ & .82 & & .75 & \\
\hline
\end{tabular}

sions were not attributable to the subjects' having rated sequences on all three dimensions.

\section{SUMMARY OF RESULTS OF EXPERIMENTS 1, 2, AND 3}

With one exception, forward sequences, reliably rated as more familiar, organized, and predictable than reverse sequences, were judged by both methods of estimation to be longer and to have more notes. Interactions between sequence direction and duration or numerosity were often significant, with sequence direction producing a larger effect on the longer durations and greater numerosities.

Temporal exponents of .88 to 1.08 for forward sequences and .80 to 1.06 for reverse sequences are within the range (.53 to 1.55$)$ of other magnitude estimation studies of time perception (Allan, 1983; Eisler, 1976; S. S. Stevens \& Galanter, 1957; S. S. Stevens \& Greenbaum, 1966). Numerosity exponents of .65 to .89 for forward sequences and .55 to .76 for reverse sequences may be lower than the reported range for numerosity exponents (.70 to 1.20) derived from magnitude estimates (Indow \& Ida, 1977; Krueger, 1972, 1982, 1984; Masin, 1983; Rule, 1966). The robust effect of sequence direction shown in the estimates occurs only in some of the comparisons of exponents and intercepts derived from verbal estimates. Although both the reliable effect of sequence direction and the interaction between sequence direction and duration or number obtained for the estimates suggest that forward and reverse sequences do differ in psychophysical functions, individual power function exponents and intercepts determined for each subject do not confirm this consistently.

Exponents and intercepts were not independent of each other. Correlations between exponents and intercepts were negative and reliable in 19 of 20 instances.

\section{DISCUSSION}

Melodies that were judged to be more familiar, predictable, and organized were estimated to be longer in duration and to have more notes than their reverse counter- 
parts, using two different, but related, methods of measurement, two different experimental designs, and different orders of stimulus presentation. Although contradictory to some reports (Block, 1978, Experiment 2; Buffardi, 1971; Cantor \& Thomas, 1977; Craig, 1973; Fraisse, 1963; Frankenhaeuser, 1959; A. Jones \& MacLean, 1966; Mo, 1971, 1974, 1975; Ornstein, 1969; Poynter \& Homa, 1983; Schiffman \& Bobko, 1977; Thomas \& Brown, 1974; von Sturmer, 1966; Vroon, 1970), these results are consistent with others (Block, 1974, Experiment 2, 1978, Experiment 1; Bobko et al., 1977; Hogan, 1975; E. C. Jones \& Natale, 1973; Kowal, 1976; Mo \& Michalski, 1972; M. J. Smith, 1975; N. C. Smith, Jr., 1969; Thomas \& Weaver, 1975; Warm, Greenberg, \& Dube, 1964; Warm \& McCray, 1969; Yeager, 1969).

These results can be explained easily by various current theories. Consider, for example, a model in which time perception varies with the number of perceived or remembered events. Our data show that subjects judge familiar melodies to have more notes (although we still only assume that more notes are remembered from the familiar than from the unfamiliar sequences) and that, as Fraisse (1963) argued but did not demonstrate, the perceived number of events rather than the actual number should determine experienced duration.

Perhaps, however, the effects of familiarity, complexity, and predictability on time judgments depend in an important way on the nature of the stimulus sequence. In many cases that agree with the present study, intervals were filled with meaningful events that had some inherent temporal structure. For example, in several studies that used words, (1) when presidents' last names were interspersed in noun lists in an unsegmented fashion, duration estimates were shorter than when the lists were segmented (Poynter, 1983); (2) intervals filled with words organized by semantic category were remembered as being longer in duration than those in which the words were in random order (Block, 1974); (3) intervals filled with frequently used words appeared to be longer than those with lower frequency words (Warm \& McCray, 1969); and (4) related-word discourses seemed to be longer than the same words in random order (Hawkins \& Tedford, 1976). Because both language and music have an inherent temporal structure, the apparent duration of segments of language or music might be contaminated by that structure in at least two ways: First, the overriding temporal structure of language or music might force the estimator to rely on that structure, thus masking the possible effects of other organizational determinants; second, a temporal structure, such as rhythm, might force the estimator to rely on a nontemporal mediator, such as counting events, when making temporal judgments. With respect to the first, we might be able to assess the effect of inherent temporal structure of event sequences on apparent duration by controlling rhythm and number of events within a sequence while varying the degree to which individual sequence items possess some inherent temporal structure. With respect to the second, a potential mediator perceived number of events-received some support in the current series of studies. Without a detailed understanding of issues such as these, no predictions about time perception are possible.

The applicability of Stevens's power law to these continua is not discredited by the present results in that temporal and numerosity exponents were similar to previously measured values for both continua. However, although sequence direction strongly affected the subjects' duration and numerosity estimates, the effect was not reflected consistently in either the exponents or the intercepts (log scaling factors) derived from estimates. Such differences in exponents that did occur were primarily in singlecontext settings (only forward or only reverse sequences were judged) and were restricted to the method of verbal estimation. The sensitivity of exponents to interval filling under these conditions could be explained as follows: The estimator mechanism whose parameters are reflected in exponent values may be able to operate differently in different contexts, but may be unable to shift its operation from one context to another within the same setting, such as when forward and reverse sequences are interspersed (dual-context setting). Differences that occur when the two contexts or types of sequences are kept separate would disappear in dual-context settings. That exponents derived from verbal estimates are affected by sequence direction, although intercepts are not, is understandable if one assumes that with verbal estimation there is homogeneity among individual scaling factors because of the subject's reliance on common units of measurement, which means common intercepts. Differences in estimates would then appear as differences in exponents. With magnitude estimation, however, fluctuations in individual scaling factors, or intercepts, could mask differences in exponents. If it is assumed that intercepts and exponents are inversely related and that intercepts differ among individual subjects, the larger magnitude estimates of forward sequences could appear in the power function in either of two ways-higher intercepts and lower exponents for some subjects or higher exponents and lower intercepts for others. Thus, no apparent effect of sequence direction on either the exponent alone or the intercept alone would be observed.

Indeed, the exponent and intercept should both be considered in describing the scaling of duration or numerosity, as the following results suggest: the failure of magnitude estimation to detect exponent differences, the pervasive significant inverse relationship between exponents and intercepts, and the observation that exponents of forward sequences are higher among subjects who have lower forward intercepts but exponents of forward sequences are lower among subjects who have higher forward intercepts. There is evidence that intercept values fluctuate in an orderly and possibly meaningful fashion (J. C. Stevens, 1974). As early as 1965 , Thalmann reported that, when subjects with normal hearing were compared with subjects suffering from a conductive hearing loss, exponents of the power function did not differ, but that intercepts for the subjects with the hearing loss were 
lower than those for the normal subjects. Thalmann suggested that intercept differences be used for diagnostic purposes. Butler and Overshiner (1983) found that intercepts increased and exponents decreased as the number of multiplications increased in the psychophysical power function relating estimated multiplication answers to the number of multiplications required by the problem. Ferguson and Martin (1983) reported that, for temporal estimates of events that occurred up to 60 months ago, the range of possible response alternatives affected both the exponent and the intercept of Stevens's law, with smaller ranges producing smaller exponents and higher intercepts. According to Algom and Cohen-Raz (1984), when magnitude estimates of velocity were obtained as a function of velocity, with either distance or duration as a parameter, in both cases the resulting exponents and intercepts of Stevens's power law varied systematically: As duration increased or distance decreased, velocity exponents increased and intercepts decreased. For the data published by Algom and Cohen-Raz, we calculated correlation coefficients relating exponent and intercept values and found that this relationship was reliable and inverse when distance was varied and duration was held constant $[r(8)=$ $-.98, p<.001]$, and when duration was varied and distance was held constant $[r(8)=-.99, p<.001]$. In all of these studies, then, increases in the exponent were accompanied by decreases in the intercept, or vice versa, which implies that a description of the scaling behavior of individuals may require specification of the value of the intercept as well as that of the exponent. Regression toward the mean might explain the inverse relationship between exponents and intercepts, as an anonymous reviewer suggested, but to evaluate that would require a different line of investigation.

The exponent provides information about the effect of stimulus factors on the perception of duration and numerosity, but only in single-context settings and with the method of verbal estimation. In any case, the inverse relationship between exponents and intercepts (log scaling factors) uncovered here shows the danger in taking one parameter as a description of subjects' judgments without considering the other.

\section{REFERENCES}

Algom, D., \& CoHEN-RAz, L. (1984). Visual velocity input-output functions: The integration of distance and duration onto subjective velocity. Journal of Experimental Psychology: Human Perception \& Performance, 10, 486-501.

Allan, L. G. (1983). Magnitude estimation of temporal intervals. Perception \& Psychophysics, 33, 29-42.

AVANT, L. L., LYMAN, P. J. (1975). Stimulus familiarity modifies perceived duration in prerecognition visual processing. Joumal of $E x-$ perimental Psychology: Human Perception \& Performance, 1, 205-213.

BLock, R. A. (1974). Memory and the experience of duration in retrospect. Memory \& Cognition, 2, 153-160.

BLOCK, R. A. (1978). Remembered duration: Effects of event and sequence complexity. Memory \& Cognition, 6, 320-326.

Bobko, D. J., Schiffman, H. R., Castino, R. J., \& Chiappetta, W, (1977). Contextual effects in duration experience. American Journal of Psychology, 90, 577-586.
BuFfardi, L. (1971). Factors affecting the filled-duration illusion in the auditory, tactual, and visual modalities. Perception \& Psychophysics, 10, 292-294.

ButLer, D. L., \& Overshiner, C. (1983). The role of mental computations in judgments of area and volume. Perception \& Psychophysics, 34, 593-598.

Cantor, N. E., \& Thomas, E. A. C. (1977). The control of attention in the processing of temporal and spatial information in complex visual patterns. Journal of Experimental Psychology: Human Perception \& Performance, 3, 243-250.

CARLSON, V. R., Feinberg, I. (1968). Individual variations in time judgement and the concept of an internal clock. Joumal of Experimental Psychology, 77, 631-640.

CraIG, J. C. (1973). A constant error in the perception of brief temporal intervals. Perception \& Psychophysics, 13, 99-104.

EISLER, H. (1976). Experiments on subjective duration 1868-1975: A collection of power function exponents. Psychological Bulletin, 83, 1154-1171.

Ferguson, R. P., \& MARTin, P. (1983). Long-term temporal estimation in humans. Perception \& Psychophysics, 33, 585-592.

Fraisse, P. (1963). The psychology of time. New York: Harper \& Row.

Frankenhaeuser, M. (1959). Estimation of time: An experimental study. Stockholm: Almqvist \& Wiksell.

Gescheider, G. A. (1976). Psychophysics method and theory. Hillsdale, NJ: Erlbaum.

Goldner, J., REUder, M. E., RibA, B., \& JARMon, D. (1971). Neutral vs. ego-orienting instructions: Effects on judgments of magnitude estimation. Perception \& Psychophysics, 9, 84-88.

HAWkins, M. F., \& TEDFord, W. H., JR. (1976). Effects of interest and relatedness on estimated duration of verbal material. Bulletin of the Psychonomic Society, 8, 301-302.

Hogan, H. W. (1975). Time perception and stimulus preference as a function of stimulus complexity. Journal of Personality \& Social Psychology, 31, 32-35.

Indow, T., IDA, M. (1977). Scaling of dot numerosity. Perception \& Psychophysics, 22, 265-276.

Jones, A., \& MacLeAN, M. (1966). Perceived duration as a function of auditory stimulus frequency. Journal of Experimental Psychology, 71, 358-364.

JonEs, E. C., \& NATALE, T. E. (1973). Information-processing theory of time estimation. Perceptual \& Motor Skills, 36, 266.

Kowal, K. H. (1976). Apparent duration of long meaningful events and meaningless intervals. Memory \& Cognition, 4, 215-220.

KowAL, K. H. (1981). Growth of apparent duration: Effect of melodic and non-melodic tonal variation. Perceptual \& Motor Skills, 52, 803-817.

KowaL, K. H. (1984). Familiar melodies seem shorter, not longer, when played backwards: Data and theory. In J. Gibbon \& L. Allan (Eds.), Tining and time perception: Annals of the New York Academy of Sciences, 423, 610-611.

Krueger, L. E. (1972). Perceived numerosity. Perception \& Psychophysics, 11, 5-9.

KRUEGer, L. E. (1982). Single judgments of numerosity. Perception \& Psychophysics, 31, 175-182.

Krueger, L. E. (1984). Perceived numerosity: A comparison of magnitude production, magnitude estimation, and discrimination judgments. Perception \& Psychophysics, 35, 536-542.

Masin, S. C. (1983). Null effect of intramodal stimulus-range variation on the exponent for numerousness. Perceptual \& Motor Skills, 56, 851-855.

Michon, F. A. (1965). Studies on subjective duration: II. Subjective time measurement during tasks with different information content. Acta Psychologica, 24, 205-219.

Mo, S. S. (1971). Judgment of temporal duration as a function of numerosity. Psychonomic Science, 24, 71-72.

Mo, S. S. (1974). Comparative judgment of temporal duration as a function of numerosity. Bulletin of the Psychonomic Society, 3, 377-379.

Mo, S. S. (1975). Temporal reproduction of duration as a function of numerosity. Bulletin of the Psychonomic Society, 5, 165-167.

Mo, S. S., MichalSKI, V. A. (1972). Judgment of temporal duration of area as a function of stimulus configuration. Psychonomic Science, 27, 97-98. 
Ornstein, R. E. (1969). On the experience of time. Middlesex, England: Penguin.

POYNTER, W. D. (1983). Duration judgment and the segmentation of experience. Memory \& Cognition, 11, 77-82.

Poynter, W. D., \& Homa, D. (1983). Duration judgment and the experience of change. Perception \& Psychophysics, 33, 548-560.

RULE, S. J. (1966). Subject differences in exponents of psychophysical power functions. Perceptual \& Motor Skills, 23, 1125-1126.

Schiffman, H. R., \& BobKo, D. J. (1974). Effects of stimulus complexity on the perception of brief temporal intervals. Joumal of $E x$ perimental Psychology, 103, 156-159.

SchiffMAN, H. R., \& BobKo, D. J. (1977). The role of number and familiarity of stimuli in the perception of brief temporal intervals. American Journal of Psychology, 90, 85-93.

SMITH, M. J. (1975). Changes in judgment of duration with different patterns of auditory information for individuals confined to bed. Nursing Research, 24, 93-98.

SMITH, N. C., JR. (1969). The effect on time estimation of increasing the complexity of a cognitive task. Journal of General Psychology, 81, 231-235.

Stevens, J. C. (1974). Families of converging power functions in psychophysics. In H. R. Moskowitz, B. Scharf, \& J. C. Stevens (Eds.), Sensation and measurement: Papers in honor of S. S. Stevens (pp. 157165). Dordrecht, Holland: Reidel.

Stevens, J. C., \& Stevens, S. S. (1963). Brightness function: Effects of adaptation. Journal of the Optical Society of America, 53, 375-385.

Stevens, S. S. (1957). On the psychophysical law. Psychological Review, 64, 153-181.

Stevens, S. S. (1966). Power-group transformations under glare, masking, and recruitment. Journal of the Acoustical Society of America, 39, 725-735.

Stevens, S. S., \& GaLANTER, E. H. (1957). Ratio scales and category scales for a dozen perceptual continua. Journal of Experimental Psychology, 54, 377-411.

Stevens, S. S., \& Greenbaum, H. B. (1966). Regression effect in psychophysical judgment. Perception \& Psychophysics, 1, 439-446.

Teghtsoonian, R., \&eghtsoonian, M. (1978). Range and regression effects in magnitude scaling. Perception \& Psychophysics, 24, 305-314.

ThalmanN, R. (1965). Cross-modality matching in a study of abnormal loudness functions. Laryngoscope, 75, 1708-1726.

Thomas, E. A. C., \& Brown, I. (1974). Time perception and the filledduration illusion. Perception \& Psychophysics, 16, 449-458.

Thomas, E. A. C., \& Weaver, W. B. (1975). Cognitive processing and time perception. Perception \& Psychophysics, 17, 363-367.

Von STURMER, G. (1966). Stimulus variation and sequential judgment of duration. Quarterly Journal of Experimental Psychology, 18, 354-357.

VRoON, P. A. (1970). Effects of presented and processed information on duration experience. Acta Psychologica, 34, 115-121.

WARM, J. S., GreenberG, L. F., \& Dube, G. S. (1964). Stimulus and motivational determinants of temporal perception. Journal of Psychology, 58, 243-248.

WARM, J. S., \& MCCRAY, R. E. (1969). Influence of word frequency and length on the apparent duration of tachistoscopic presentations. Journal of Experimental Psychology, 79, 56-58.

YEAGER, J. (1969). Absolute time estimates as a function of complexity and interruption of melodies. Psychonomic Science, 15, 177-178.

ZwISLOCKI, J. J., \& GoOdMAN, D. A. (1980). Absolute scaling of sensory magnitudes: A validation. Perception \& Psychophysics, 28, 28-38.

(Manuscript received August 5, 1985; revision accepted for publication February 17, 1987.) 